\title{
Inspiring Responsive Interior Architecture from the Inherent Hygroscopic Behavior of Pine Cones
}

\author{
Loka S. El-Darir1, Dalia S. Alabassy¹, Suzan H. El-Gazar², Lubna S. El-Darir ${ }^{1}$ \\ ${ }^{1}$ Interior Design, Faculty of Fine Arts, Alexandria University, Alexandria, Egypt \\ ${ }^{2}$ Interior Design, Faculty of Arts and Design, Pharos University, Alexandria, Egypt \\ Email:lokaeldarier@yahoo.com,Dalia_alabassy@yahoo.com,Suzan.hassan@Pua.edu.eg,lubna.eldarier@gmail.com
}

How to cite this paper: El-Darir, L.S., Alabassy, D.S., El-Gazar, S.H. and El-Darir, L.S. (2019) Inspiring Responsive Interior Architecture from the Inherent Hygroscopic Behavior of Pine Cones. Journal of Biosciences and Medicines, 7, 173-183.

https://doi.org/10.4236/jbm.2019.711016

Received: November 5, 2019

Accepted: November 26, 2019

Published: November 29, 2019

\begin{abstract}
Architectural Engineering has experienced a new trend that incorporates Synthetic Biology Concepts and expressions. This trend is not only limited to borrowed terminology, but also extends to embrace new types of construction processes derived from living organisms' actuating properties. The linkage between biology, architectural engineering and interior design could restructure the current physical applications of materials used in design to give new forms and patterns inspired from Plants behavior with efficient and adaptive characteristics. As a result of the growing need for responsive new design models to more flexibility between design process and the attributed fundamental properties of materials, there is a strong relationship between design and the composition of materials. This can be achieved by co-evolutionary design strategy, already applied in synthetic biology, by applying computational simulations to express the behavior of Plants to set a computational tool based on morphological and biological principles. Natural actuators, like Pine cones, have the ability to move their scales upon humidity gradient; as this, Hygroscopic behavior gives substantial stability to the inner relative humidity. In this paper, the importance of "hygroscopic behavior of Pine cones" is analyzed in designing spaces that actuate to generate responsive morphological patterns where different wood layers begin to react. A pavilion was designed in accordance with the actuation in plant organs which respond to changes in environmental humidity. We also present the results of an experimental study that was conducted on different humidity ranges. The goal of this study is to achieve a new level of studies on the biomimetic transfer of the plant motion principles into movable technical systems.
\end{abstract}

\section{Keywords}

Hygroscopic Behavior, Hygroscopic Actuators, Responsive Architecture, 3D-Printed Wood 


\section{Introduction}

SELF-ORGANIZATION is a process through which the internal organization of the system adapts to the environment to promote a specific function without being controlled from outside. Biological systems have adapted and evolved into efficient configurations, which are symbiotic with the environment [1].

The premise of this research is to integrate sensing and actuation functions into a composite material system that gives the possibility for local variations in their material properties which could be used to sense multiple parameters.

\section{The Main Concepts}

The ideas used to express the organized relationship between the parts of the biological organism like wholeness, coherence, correlation and integration; can be applied to describe similar qualities in the well-designed artifact. The adaptation and fitness of the organism to its environment can be compared to the harmonious relation of a building to its surroundings [2].

Adjustable plant movements are advantageous for being energy efficient and integrative, where many of these advantages originate from strategic combinations of various actuation mechanisms and physiological features (Figure 1).

\section{Biological Inspirations in Technique and Technology}

\subsection{Biomimetic Responsive Surface Structures}

Anisotropy and hygroscopy in nature are employed to exploit dimensional changes aroused by changing climatic conditions in order to stimulate shape changes to reactive material elements (Figure 2). Material systems in plants in combination with hygroscopic properties can also perform kinematic and

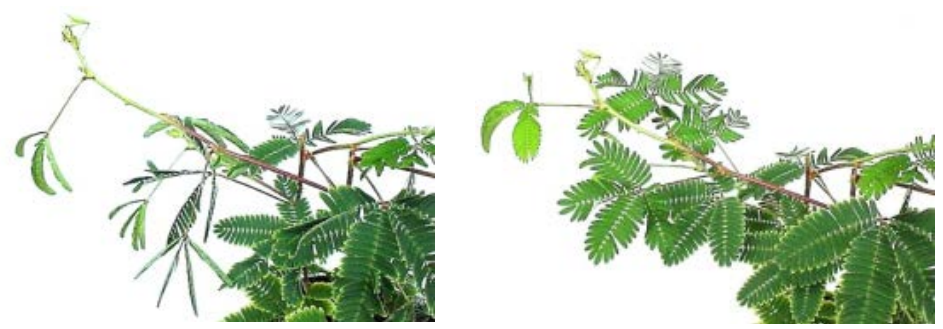

Figure 1. Mimosa pudica plant, which closes its leaves when lightly touched.

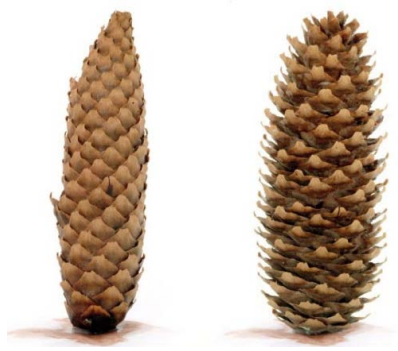

Figure 2. The biological principle of changing the shape of Pine cones by adapting to their environment. 
environmentally responsive movements without the use of muscles, beside making use of an anisotropic material set up to adapt to structural forces. Therefore, these systems provide a new conceptual and practical framework for truly environmentally responsive architectural surface structures, which enables the design and manufacturing of climate responsive surface structures with no need for any additional mechanical or electronic control [1].

\subsection{Hygroscopic Morphology}

Hygroscopy is the phenomenon of absorbing water molecules from the surrounding environment.

*hy · gro cop · ic (Adj.) Tending to absorb moisture from the atmosphere.

${ }^{*}$ mor $\cdot$ phol $\cdot \mathrm{o} \cdot \mathrm{gy}$ (n.) a. The branch of biology that studies the form and structure of organisms without consideration of function.

In nature, a wide range of dynamic systems interact with climate effects. The moisture-driven motion that can be observed in Pine cones seems to be a particularly exciting way for architecture. This motion takes place through a passive response to changes in humidity. No energy is consumed by the motion because it is free of any metabolic function. Anisotropy indicates the directional dependence of material features. Hygroscopicity indicates the potential of a substance to absorb moisture from the atmosphere when dry and release moisture to the atmosphere when wet, thus keeping a content of moisture in balance with the relative humidity surrounding it.

The movement of Pine cones is based on the actual ability of the material to interact with the external environment and demonstrates how a structured tissue can respond passively to environmental changes: cone opening (once dried) and closing (once wetted). The outer layer, formed of parallel, long and tightly packed thick-walled responds cells, hygroscopic ally reacts to an increase or decrease in humidity levels by expanding or contracting, while the inner layer stays comparatively stable (Figure 3 ).

In architectural context, hygroscopic morphology is known as the study of forms made from materials that absorb moisture from air. The main goal is to generate kinetic energy without any artificial mechanical assistance.

\subsection{Strategies of Using Material Anisotropy to Achieve Sophisticated Hygroscopic Deformation}

\subsubsection{Bimorph}

A bimorph consists of two layers of distinct materials: frequently one layer is active and can swell and shrink as a result of changes in atmospheric humidity, and the other layer is passive and acts as a kinematic restriction (Figure 4) [3].

\subsubsection{Multi-Material Modulation}

Bimorph can only bend, but more complicated deformations such as twisting require more advanced material allocations by combining stripes of two constituent materials at a prescribed orientation (Figure 5) [3]. 


\subsubsection{Fiber Reinforcement}

Composites of hygroscopic fiber closely resemble fibrous plant tissues. The deformed shapes and elastic characteristics of the composites are directly related to the orientations of these reinforcing fibers (Figure 6) [3].

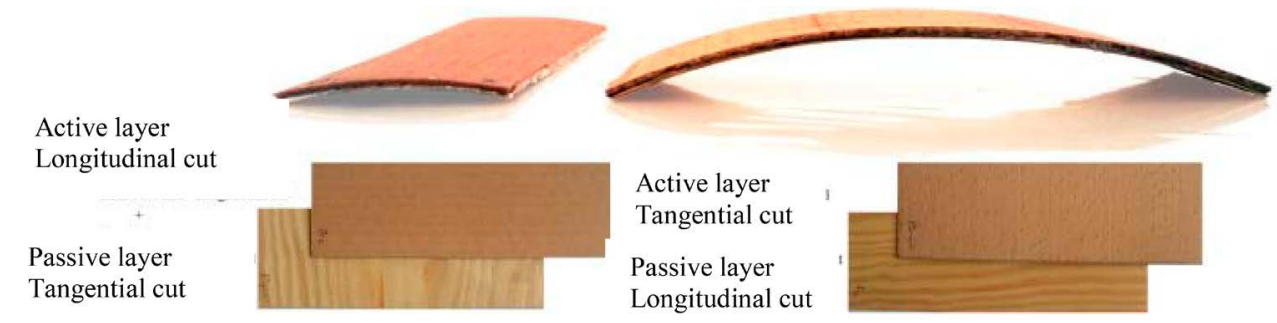

Figure 3. Hygroscopic behavior of thin Pine Veneer material.
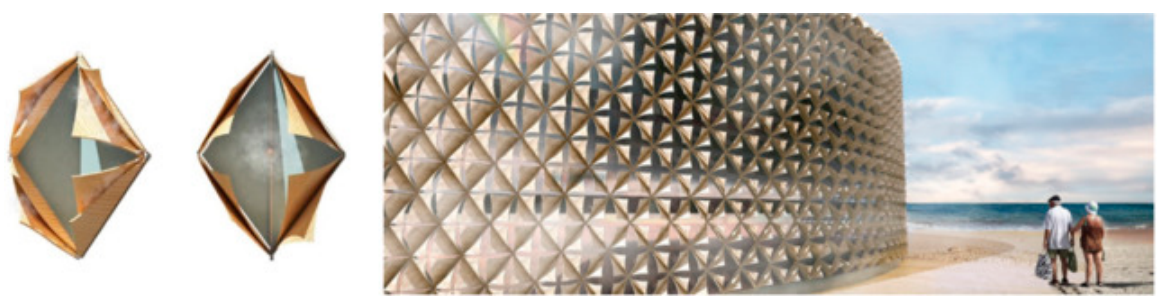

Figure 4. Bimorph implemented material as responsive building skins.

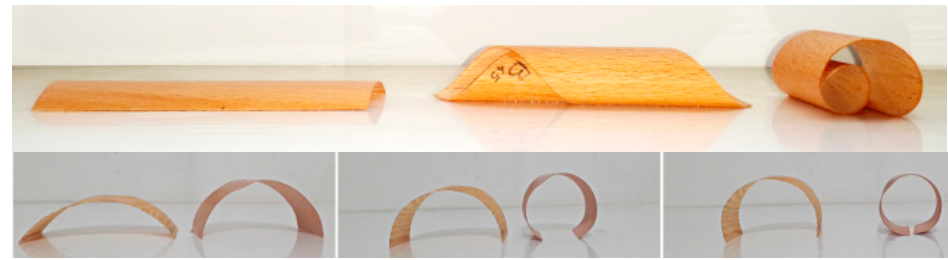

Figure 5. Material modulation for helical twisting.

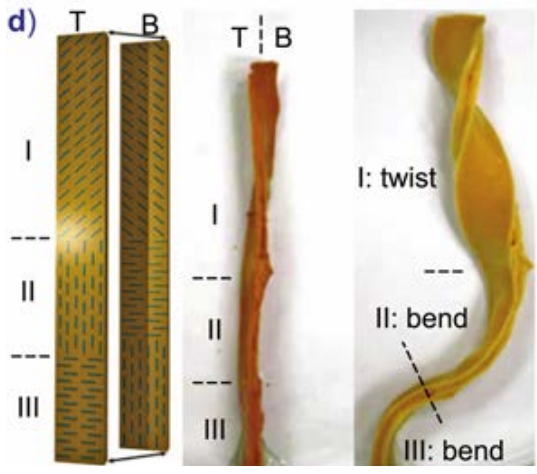

Figure 6. Programmed short fiber reinforcement for complicated deformation.

\section{Methodology of Design Inspired from Biology}

Hygroscopic morphology is considered more than the kinetic energy generated from particular materials like wood. We decided to focus on combining these hygroscopic behaviors and creating some kind of mechanism that could be responsive to humidity. A kinetic Pavilion that would adapt to the humidity condi- 
tions by changing its shape.

\section{Practical Application}

Inspired by the dynamic hygroscopic conduct of the pine cone, the research focuses on the use of the dimensional change of hygroscopic components of the $3 \mathrm{D}$ printed material system to start a shape change in response to fluctuations of external relative humidity [4]. For creating responsive smart structures, wood swell and shrink consider a precious material.

It was inspired from the responsive behavior of various parts of the plant, such as pine Cones opening and closing. The suggested model for practical application examines the essential behavior of multilayered timber to be used in interior design as responsive components. Differential fiber orientation causes differences in bending and reverse bending dimensional modifications. By understanding basic design principles, the designer can create biologically transformable components as a consequence of daily modifications in air humidity or exposure to rain without any extra mechanical or electronic control [5]. By using multimaterial 3D printing techniques and anisotropic material compositions, hygroscopic material such as wood can be physically programmed to accurately sense, and self-transform based on environmental changes [6].

This Pavilion Design seeks to improve the anisotropic and hygroscopic characteristics of wood by both designing and 3Dprinting custom wood grain structures to support controllable auto-transformation. The methods, techniques and material trials illustrated outcomes of differentiated printed wood for auto-transformation behavior, proposing a new strategy for programmable material and responsive architecture [5].

\subsection{Wood Activation Techniques}

The 3Dprinted wood composites (both wood or multimaterial) must be subjected to moisture to cause wood transformation: direct water submersion, dry or moist air (R.H.) through a climate chamber, and heat radiation coupled with one of the moisture intake techniques [5].

\subsubsection{Water Submersion}

The first moisture intake technology uses the full water submersion of the composite of printed wood. The printed samples are placed inside a water vessel at room temperature until full saturation of the wood composite.

\subsubsection{Water Vapor}

This procedure can be used to subject the samples from very dry to a full range of relative humidity to remove moisture or very moist in order to boost moisture in the print.

\subsubsection{Thermal Radiation}

To activate transformation, thermal radiation may be combined with either the water vapor or the water submersion technique. Thermoplastics can have large 
changes in their physical properties due to heat at various temperatures.

\subsection{Methods for 3D-printed Wood Transformation}

Method A: differentiated printing (wood-only). Through 3D printing (Figure 7, Figure 8).

Differentiated printing of wood relies on customized deposition of wood filament over many layers. The transformation is caused by the natural expansion and contraction of the fibers due to water absorption. Under controlled humidity and/or heating conditions, the material can be programmed to generate distinct curling or folding deformations [5].

Method B: differentiated multi material printing (Figure 9, Figure 10).

Differentiated multimaterial printing continues to increase on the previous method by using two materials, a reactive material with a high hygroscopic swelling ability (wood fiber composite) and a non-reactive material. The difference in volumetric expansion, bending stiffness and elasticity modulus of each layer forms the basis for the responsive deformation behavior, and modulus of elasticity of each layer. 3D printing is used in this method as a way to create custom-differentiated multilayer composites [5].

\subsection{Proposal Pavilion Design}

Figure 11 shows the stages of building the initial hygroscopic form of the unit

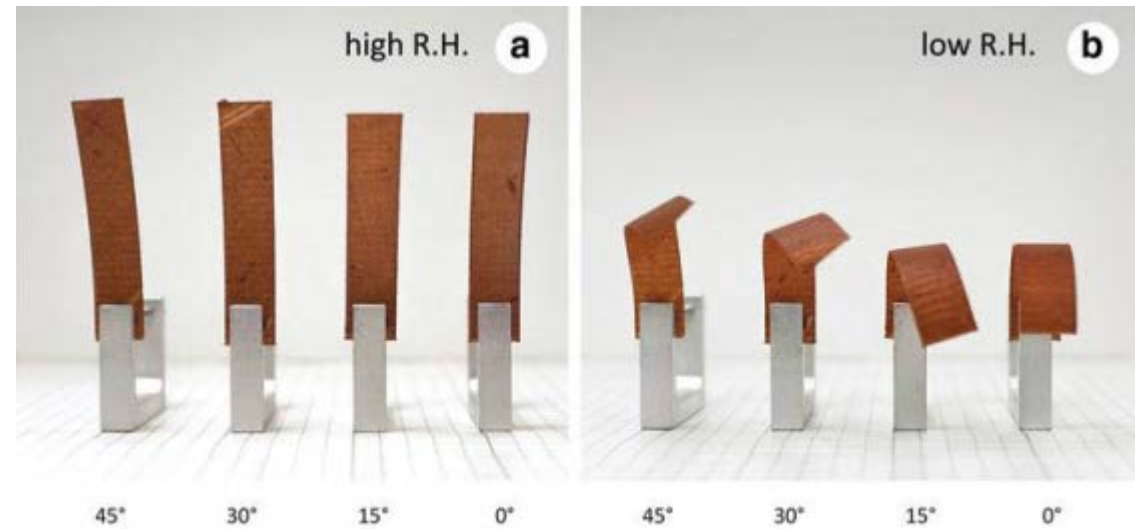

Figure 7. Controlled curling angles based on 3D-printed, Samples with no deformation under high R.H. (left, a) and high deformation under low R.H. (right, b).
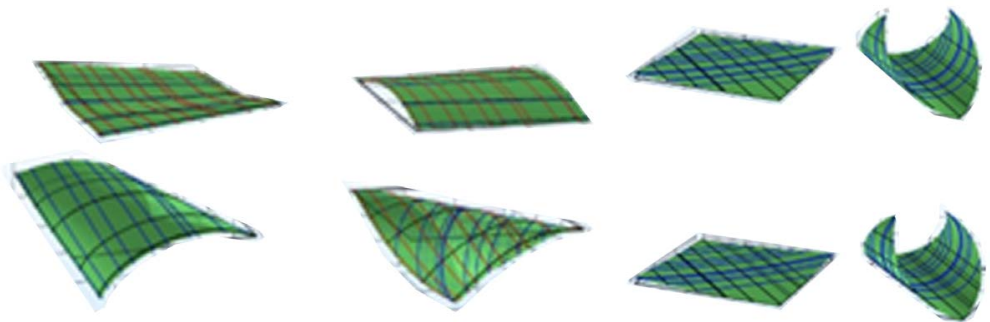

Figure 8. A series of photographs showing a differentiated self transforms printed wood composite. 


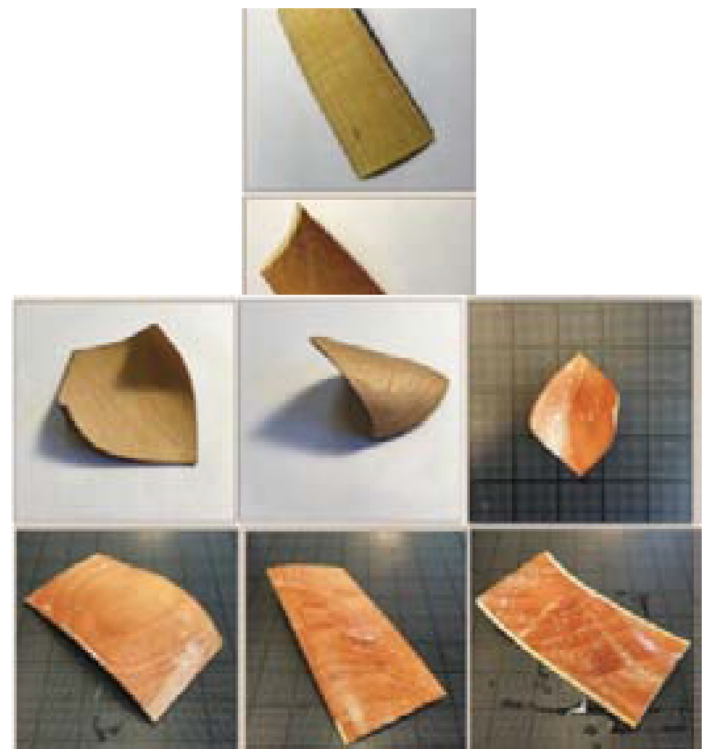

Figure 9. A series of photographs showing a multimaterial printed wood composite that self-transforms from a flat sheet into a symmetrically folded structure.

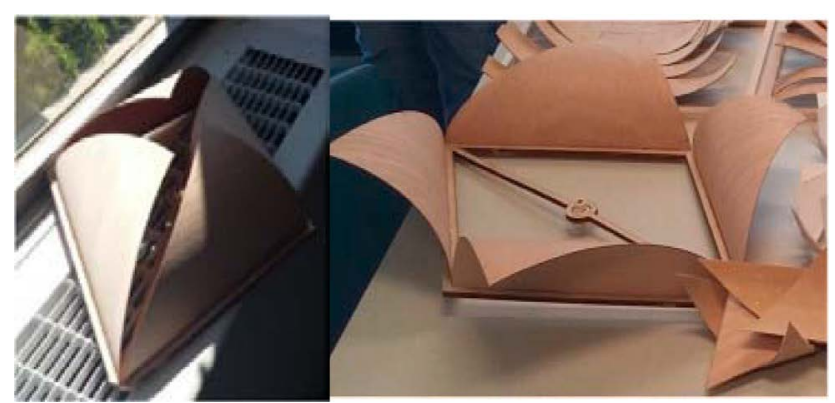

Figure 10. Photo of single-material 3D-printed test sample.

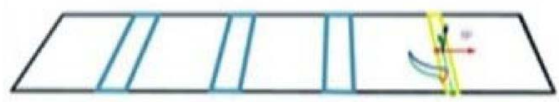

Determination of the responsive surface

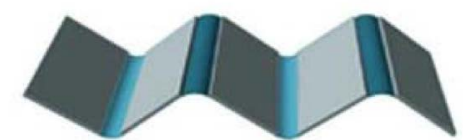

Simulation: In case of low air humidity

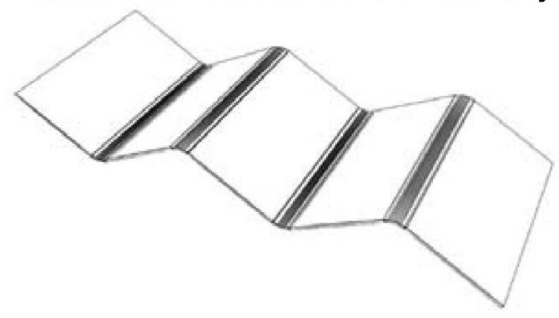

Figure 11. Stages of building the initial hygroscopic form of the unit used in the design, to determine both the active and passive space of the wooden surface to control the resulting movement. 
used in the design, to determine both the active and passive space of the wooden surface to control the resulting movement.

Figure 12 shows the plan and Elevation of the Responsive Wooden Strips used in the Pavilion's Design.

Figure 13 shows the vertical Section of the wooden sheet's layers used in design.

Figure 14 shows the design of wooden Stripes that computationally programmed to interact with different weather factors through space positions variance. Figure 15 shows the vertical-side projections of the three movements proposed for the Pavilion design according to the humidity change degree.

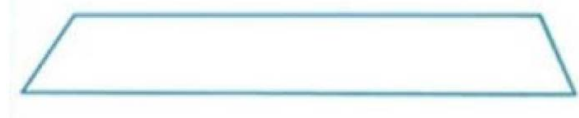

Determination of the effective material surface

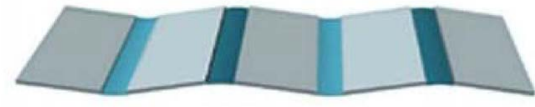

Simulation: In case of high air humidity

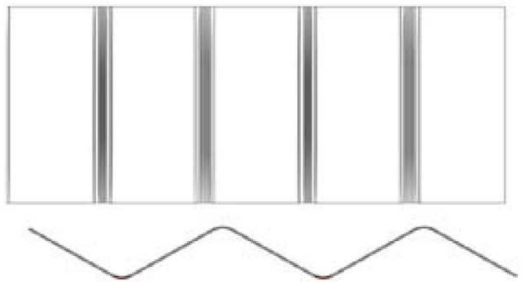

Figure 12. Plan and Elevation of the Responsive Wooden Strips used in the Pavilion's Design.

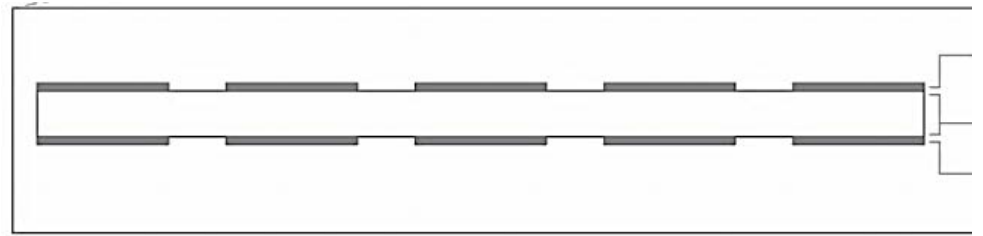

Figure 13. Vertical Section of the wooden sheet's layers used in design.

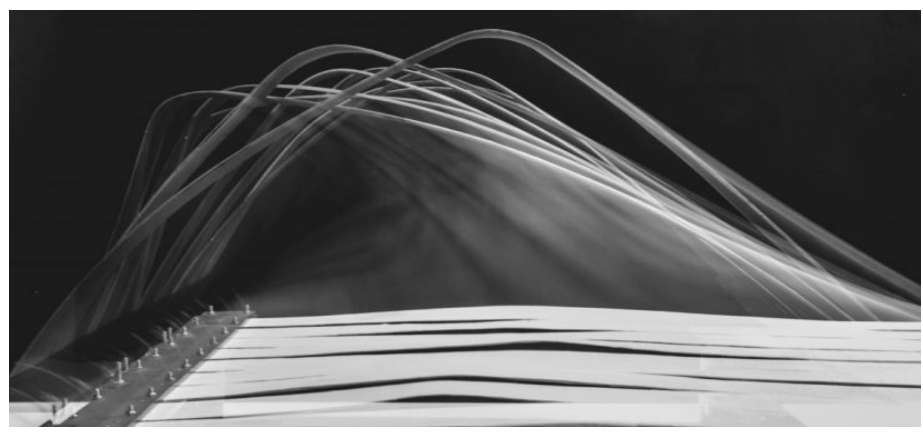

Figure 14. Design of wooden Stripes that computationally programmed to interact with different weather factors through space positions variance. 


\subsubsection{First Response Movement}

The first response movement indicates the first proposed configuration of the space depending on the degree of wooden tapes bending according to humidity change (Figures 16-18).

\subsubsection{Second Response Movement}

The second response movement indicates the second proposed configuration of the space depending on the degree of wooden tapes bending according to humidity change (Figures 19-21).

\subsubsection{Third Response Movement}

The third response movement indicates the third proposed configuration of the space depending on the degree of wooden tapes bending according to humidity change (Figures 22-24).

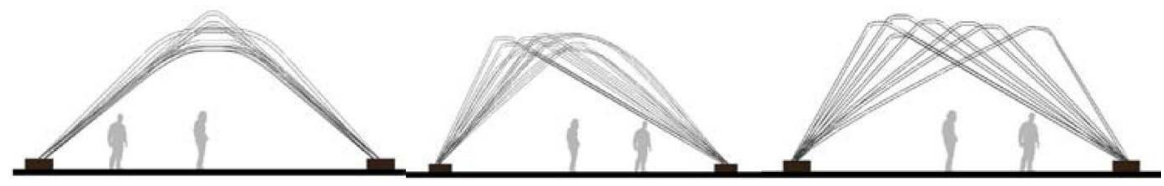

Figure 15. Vertical-side projections of the three movements proposed for the Pavilion design according to the humidity change degree.

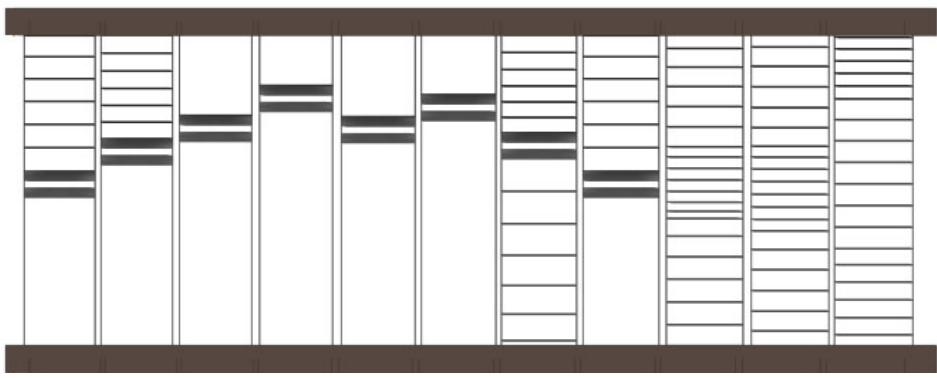

Figure 16. Pavilion Plan (First Movement).

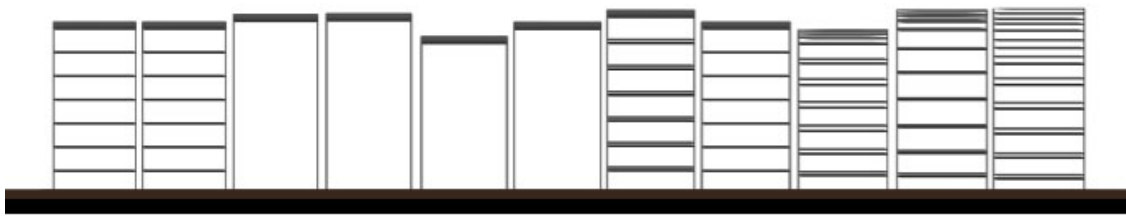

Figure 17. Pavilion Section (First Movement).

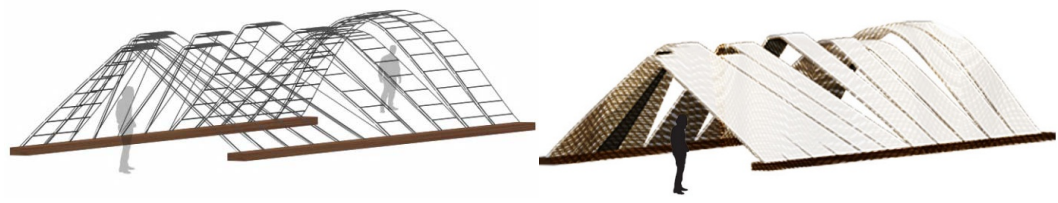

Figure 18. A perspective illustrates the different configurations of the space depending on the degree of bending of the wooden tapes according to the degree of humidity (First Movement). 


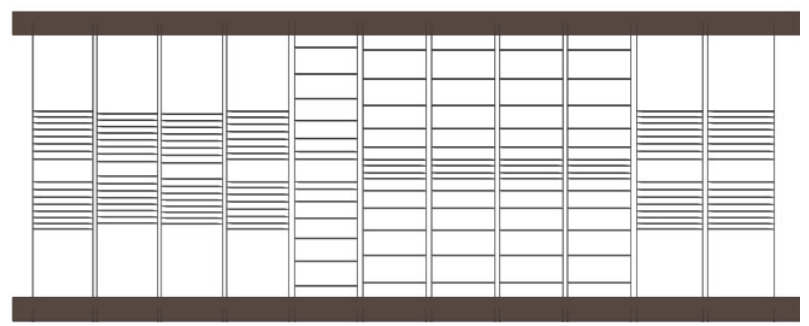

Figure 19. Pavilion plan (Second movement).

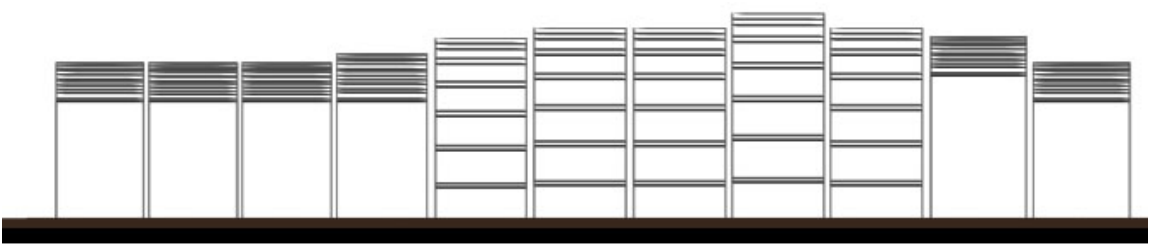

Figure 20. Pavilion Section (Second Movement).

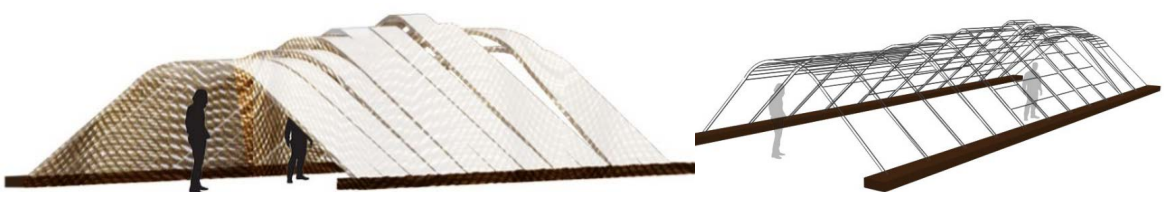

Figure 21. A perspective illustrates the different configurations of the space depending on the degree of bending of the wooden tapes according to the degree of humidity (Second Movement).

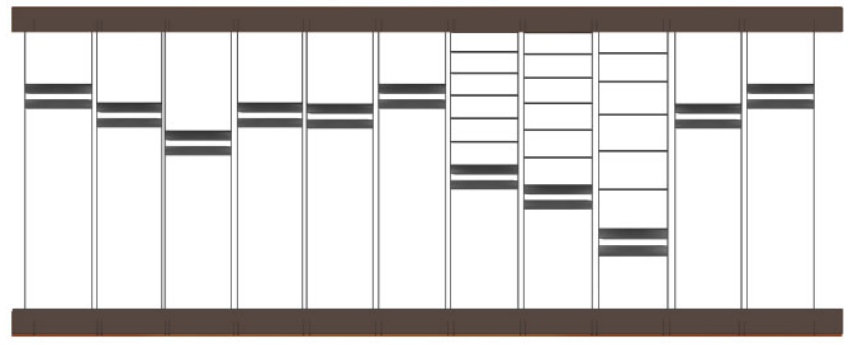

Figure 22. Pavilion plan (Third movement).

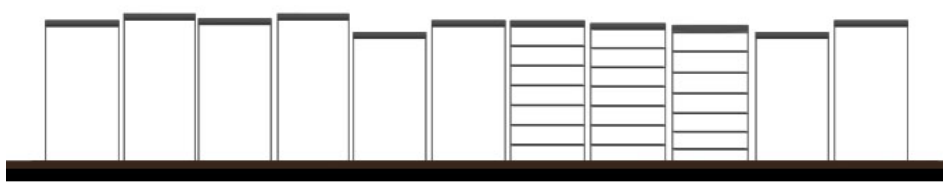

Figure 23. Pavilion section (Third movement).

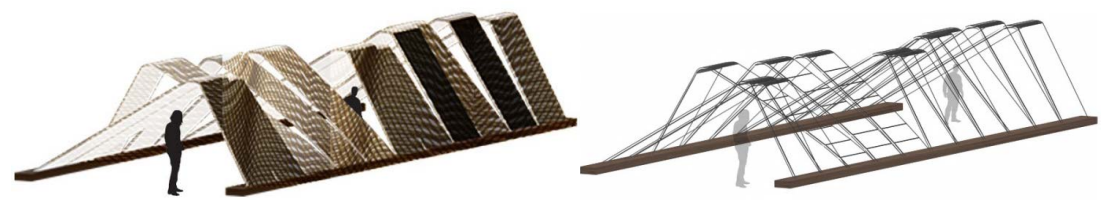

Figure 24. A perspective illustrates the different configurations of the space depending on the degree of bending of the wooden tapes according to the degree of humidity (Third Movement). 


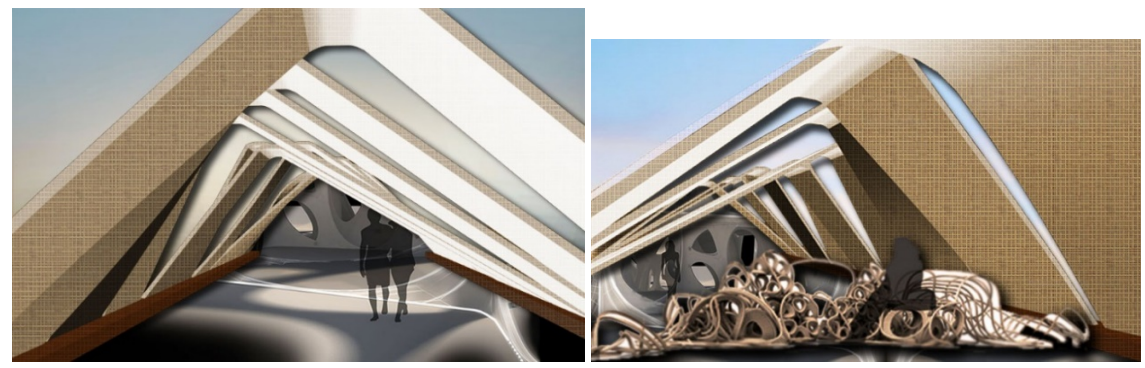

Figure 25. Interior Shots of the Responsive Pavilion that react to the external environment through applying the hygroscopic features.

\section{Conclusion}

Movements in the botanical kingdom provide us with a lot of lessons to attain distinct form and motion in an integrated and adaptive structural system and how artificial systems of various dimensions and specific applications in architecture and interior architecture were achieved (Figure 25). The conclusion reached is that this strategy includes two techniques of adaptive design:

- Design mechanisms, that use morphological parameters based on continuous dynamic, compromises between design purpose and materials' physical appearance.

- The suggested design strategy seeks to produce biologically productive forms with adaptive organisms' potential behavior.

\section{Conflicts of Interest}

The authors declare no conflicts of interest regarding the publication of this paper.

\section{References}

[1] Mingallon, M. and Ramaswamy, S. (2012) Bio-Inspired Self-Actuating Composite Materials. Open Access Peer-Reviewed Chapter, Published: August. https://doi.org/10.5772/47860

[2] Steadman, P. (1979) The Evolution of Designs: Biological Analogy in Architecture and the Applied Arts' Syndics of Cambridge University Press, P. 4.

[3] Li, S.Y. and Wang, K.W. (2017) Plant-Inspired Adaptive Structures and Materials for Morphing and Actuation: A Review. Bioinspir. Biomim, 12, 10-12. https://doi.org/10.1088/1748-3190/12/1/011001

[4] Alabassy, D. (2018) Application of Branching Systems with Digital Morphogenesis Methodology in Computational Interior Architecture. PhD Thesis, Faculty of Fine Arts, Alexandria University, Egypt.

[5] Correa, D., Papadopoulou, A., Guberan, C., Jhaveri, N., Reichert, S., Menges, A. and Tibbits, S. (2015) 3D-Printed Wood: Programming Hygroscopic Material Transformations. 3D Printing and Additive Manufacturing, 2, 106-111. https://doi.org/10.1089/3dp.2015.0022

[6] Institute for Computational Design, University of Stuttgart, Stuttgart, Germany. 